\title{
LA CONSTRUCCIÓN DEL RELATO MÍTICO ANCESTRAL EN EL ARTE Y LA POESÍA MAPUCHE ACTUAL. ${ }^{1}$
}

\begin{abstract}
Mabel GARCÍA BARRERA ${ }^{2}$
\section{Resumen.}

El arte mapuche actual se caracteriza por mezclar prácticas de la producción artística y del saber cultural mapuche ancestral como de la cultura occidental. En este sentido, la actual obra de arte se constituye en un topos agonístico donde se desplazan, significan y resignifican elementos y categorías, propias y ajenas, buscando definir una identidad; así, la obra se abre tanto hacia la recuperación de lo propio -a través del proceso de retradicionalización cultural-, como hacia la desvinculación de lo ajeno -a través del proceso de resistencia cultural-.

En esta dinámica, el relato mítico ancestral se ubica como uno de los elementos artísticoculturales transversales donde reafirmar la diferencia cultural y al mismo tiempo como un importante vehiculador de las bases estéticas tradicionales. Desde este punto de vista, el presente trabajo busca saber cómo el actual proceso artístico y literario mapuche resignifica el relato mítico y en este proceso transforma o no algunas de las bases estéticas inscritas en él.
\end{abstract}

Palabras claves: arte mapuche, poesía, mito, retradicionalización cultural

\begin{abstract}
.
Current Mapuche art is characterized by mixing practices of artistic production and knowledge of both Mapuche ancestral culture and Western culture. In this sense, the current Mapuche art becomes a conflicting field where their own elements and categories and the foreign ones move, signify, and resignify, in search of defining an identity; thus, the work opens both towards the recovery of their own traditions - through the cultural tradition revival process - and the separation from the foreign ones- through the cultural resistance process.

In this dynamics, the mythical ancestral narration ranks as one of the cross-cultural artistic elements where the cultural difference is reasserted and, at the same time, as an important vehicle for the traditional aesthetic bases. From this point of view, this paper seeks to know how the ongoing Mapuche artistic and literary process resignifies the mythical narration and, in this process, whether it transforms some of the aesthetic bases inscribed on it or does not.
\end{abstract}

Key words: Mapuche art, poetry, myth, cultural tradition revival

\footnotetext{
${ }^{1}$ Este trabajo forma parte del Proyecto de Investigación Diufro D109-0010 "Arte mapuche actual. Constitución de un sistema estético-cultural propio y diferenciado" que dirige la autora y se adscribe a los trabajos del Doctorado en Filosofía, con mención en Estética y Teoría del Arte de la Universidad de Chile.

${ }^{2}$ Universidad de La Frontera -Temuco- Chile mabelg@ufro.cl
}

Fecha de recepción del artículo: Octubre 2010

Fecha de evaluación: Noviembre 2010 


\section{Résumé.}

L'art des Mapuches se caractérise par la pratique mixte de la production artistique et de la connaissance culturelle ancestral Mapuche avec des éléments de la culture occidentale. En ce sens, l' œuvre d'art actuelle s'établit dans un lieu agonistique où se déplacent, signifient et resignifient des éléments et catégories, propres et des autres, en cherchant de définir une identité; ainsi l'œuvre s'ouvre à la reprise de elle même - à travers de le processus de retraditionalisation culturelle-, comme le découplage de ce que n'est pas propre - à travers de le processus de résistance culturelle-.

Dans cette dynamique, le récit mytique ancestral se place parmi les éléments artistiquesculturelles transversaux qui sert pour réaffirmer la différence culturelle et en même temps comme un outil important des bases esthétiques traditionnelles. De ce point de vue, ce document cherche à savoir comment le processus artistique et littéraire actuel des Mapuches redéfinit le récit mytique et dans le processus transforme ou non certains bases esthétiques inscrit sur elle.

Mots clés: art mapuche, poésie, mythe, re-traditionalisation culturelle

\section{La cultura mapuche: lecturas de un viaje al tiempo anterior.}

El pueblo mapuche estableció su territorio ancestral entre el río Bío- Bío por el norte de Chile y el golfo de Reloncaví por el sur, extendiéndose de este a oeste entre el océano atlántico y el océano pacífico de América del Sur. Como todos los pueblos originarios de Latinoamérica fue despojado de este territorio e integrado a los actuales estados nacionales chileno y argentino después de una larga historia de conflictos bélicos que derivaron en la imposición de un sistema socio-político y cultural hegemónico, proceso ante el cual se ha distinguido por mantener una fuerte posición de resistencia y reinvindicación étnico-cultural anclada en la defensa de una noción cultural altamente compleja como la de "tierra-territorio"3.

$\mathrm{Su}$ cultura tradicional ancestral se caracterizó por un sistema de organización social dividido en lof -familias-, quienes tenían en su origen un antepasado común y un ser tutelar por alianza lo que los llevó a establecer un mismo centro ceremonial o "rehue"; además del reconocimiento de las líneas consanguíneas también los mapuches se reconocían a sí mismos por su pertenencia a territorios específicos: "Diversas parcialidades participaron en la construcción de una ecúmene cultural propia al interior de este territorio: moluches, nagche, lafkenches, pehuenches, pampas, renqueles, huilliches, tehuelches, regidos por el Ad Mapu mapuche" (cfr. Toledo Llacaqueo, Víctor. 2006.22)

Este pueblo, desarrolló un sistema cosmovisional a partir de una concepción sacralizada, dinámica y circular del universo, el que divide en tres dimensiones principales donde se desplazan los hombres, los antepasados y las fuerzas o entidades sobrenaturales: "Wenu mapu" o tierra de arriba, "Minche mapu" o tierra de bajo y "Nag mapu" lugar donde reside el ser humano junto a las fuerzas nouménicas, con las cuales convive y se comunica constantemente.

Para sostener estas relaciones entre los diversos planos, tanto los códigos verbales como los no verbales -entre los cuales se encontraba el mapudungun como idioma propio no gráficoactuaban en una doble función: a) transmitir/organizar información para propiciar la vinculación social; b) transmitir/organizar cifradamente el conocimiento restringido sobre las formas de comunicación, normas y dinámicas del azmapu, donde se encuentra la potenciación del "newen" -fuerzas- y rol del "küpalme" y del "tügun" de la persona. Así, la música, las iconografías, la disposición espacial del habitat, los colores, los saludos, entre otras actividades, responden a esta doble función comunicativa, la que sólo puede ser descifrada y manejada dependiendo del grado de competencia cultural que alcanzan los miembros de la cultura, ya de alguna manera inscrito en su mismo "küpalme".

\footnotetext{
${ }^{3}$ Cfr. Dichos conceptos se encuentran asociados con el "waj mapu" (cosmos) y "wajontu mapu" (expresión juridiccional del territorio como el lugar donde se encuentran las identidades territoriales)
} 
En esta concepción del universo, el tiempo referencial y significativo es el pasado, el que otorga sentido a la contingencia, "... ya que es la fuente de la información a la cual se recurre para fundamentar, apoyar o tomar decisiones, en todas las acciones socioculturales que puedan tener incidencia en el futuro de la sociedad en su conjunto" (Quilaqueo et al. 2005.107). Este proceso natural de tradicionalización constante de su cultura ancestral, vía este recorrido hacia atrás en el tiempo, quizás ha sido uno de los mecanismos más fuertes para su retroalimentación y fortalecimiento en contextos adversos, como lo fue la intervención histórica, religiosa y política de occidente.

Para el mapuche, este viaje constante en el tiempo ayuda a explicar su propia existencia en el nag mapu -la tierra visible- a partir del reconocimiento de su linaje, donde el "tuwun" -vínculo territorial- y/o el "Kvpalme" -vínculo por sangre- definen su fuerza espiritual, su rol y función comunitaria ${ }^{4}$; una lectura sobre el pasado que puede verse también aplicada en la práctica ritual llamada pentukun donde "la persona que se presenta hace una narración, remontándose al pasado hasta la cuarta generación, indicando el lugar geográfico de proveniencia, las características que presenta el lugar, la genealogía familiar...hasta llegar a decir inche "aquí estoy yo y así soy" (Quilaqueo et al.2005.81) $)^{5}$

El mito como acontecimiento complejo, verdadero, de carácter hierofánico, en este contexto surge como un elemento articulador del saber de la cultura ancestral y como una forma de actualización del pasado, encontrándose narrado en "epew" - relatos-, actualizado como práctica ritual mediante las instancias ceremoniales, representado en los lenguajes tradicionales de este pueblo: los "pewma" o sueños y el "perrimuntun" o visiones, y también en la vida cotidiana a través del encuentro y alianza con las entidades que pueblan su entorno natural.

Sobre el mito tradicional mapuche, el año 1990 el investigador Hugo Carrasco logró establecer ciertas características que permiten una mejor aproximación a él, distinguiendo algunos tipos al interior del evento hierofánico primordial a partir de la relación que se establece entre el tiempo en que se desarrollan los acontecimientos y el tipo de alianza que se da entre seres sobrenaturales y seres humanos. Esta propuesta señala cuatro tipos de relatos:

el primero, refiere al tiempo de los orígenes donde Ngenechen o Ngenemapun crean el mundo y al hombre, llamado "Mito de creación cosmogónico-antropogónico"; el segundo, refiere al tiempo de destrucciones y regeneraciones cósmicas y universales que corresponde a un pasado muy remoto, allí Tren Tren y Kai Kai producen la destrucción y renacimiento del mundo y del hombre, llamado "Mito del diluvio o de Tren Tren y Kai Kai"; el tercero, en el tiempo de la historia humana, en el pasado remoto, donde los seres sobrenaturales positivos o negativos actúan sobre los seres humanos, llamados "mitos de transformación"; y, el cuarto, también en el tiempo de la historia humana, pero sólo denominado "el pasado", donde los seres humanos se transforman en sobrenaturales al encontrarse con ellos, expresándose en mitos diversos tales como el mito de "Mankián", de "Shumpall", o el de "Chon-Chon", entre otros (cfr. Carrasco, Hugo. 1990.105).

Esta relación constante con lo sobrehumano, obliga al mapuche a estar en permanente alerta sobre sus decisiones generándose una fuerte conciencia ética sobre su relación con el medio natural y con los lazos y prácticas comunitarias para no violentar las normas del "az mapu", sobre las que señala Elicura Chihuailaf -uno de los principales poetas mapuches- "Son las normas que ordenan la reciprocidad, el espacio en el que es posible alcanzar el intercambio con el fin de otorgarle continuidad a los equilibrios duales que dicen relación, por ejemplo con el día y la noche; la salud y enfermedad; arriba y abajo; alegría y tristeza; anciano y joven; mujer y hombre; vigor y debilidad. Las Energías positivas Kvme: newen fuerza, kvrvf viento, neyen aliento, pvllv espíritu. Y las Energías negativas Weza: newen, kvrvf, neyen, pvllv.” (1999.50)

\footnotetext{
${ }^{4}$ Cfr. Por ejemplo con los roles de "lawentuchefe", "machi", "lonko" o "dungunmachife", entre otras funciones tradicionales reconocidas por la cultura.

5 "Yñey no rume kimlay cheu amutual kimnolu cheu ñi kupalme. Nadie puede saber donde ir, si no sabe de donde viene" (Manquenahuel, Juan P.)
} 
El respetar o transgredir las normas del Az Mapu tiene consecuencias para el espíritu de la persona y su linaje, no sólo de los que vendrán sino de aquellos que le antecedieron, debido a que "La vida no tiene fin en el Pueblo mapuche, solo se transforma, y es cíclica permanentemente, se repite, se transforma por los ciclos de los ciclos. No hay lecturas de fin de mundo, ni trágica ni magnífica. Nada se termina, todo se transforma y algún día vuelve a ser lo que fue en el pasado por muchos ciclos." (Ñanculef H, Juan. 2009)

\section{Arte mapuche actual. Las resignificaciones estéticas en el relato mítico ancestral.}

En el marco de un contacto cultural hegemónico y de relaciones culturales asimétricas -donde han sido históricamente coartadas las prácticas culturales ancestrales y subsumida la lengua materna, el mapudungun, a un estado de riesgo lingüístico-, a inicios de la década de los noventa el pueblo mapuche emprende un fuerte proceso de retradicionalización ${ }^{6}$ cultural. Este proceso surge como una actividad reflexiva vinculada a la reafirmación de una identidad cultural propia y diferenciada, y como parte de los procesos de resistencia cultural, cuya expresión tangible se encuentra en la recuperación de las prácticas tradicionales ceremoniales, de organización social, del sistema de comunicación ancestral así como también de la visibilización de los componentes culturales en una serie de discursos que tienen su origen en el espacio intercultural, como es el caso del discurso artístico.

El denominado "arte mapuche actual" -poesía, cuento, mural, plástica, dibujo, grabado, escultura, teatro, música, entre otros-, establece un amplio campo de producciones artísticas surgidas en el espacio de contacto cultural con la sociedad global, las que adquieren una dimensión simbólica al vincularse con interrogantes profundas sobre los procesos epistemológicos, estéticos, éticos y ontológicos de un pueblo que aspira a rearticular su ethos cultural ancestral tradicional en una dinámica histórico-política hegemónica y socioculturalmente cambiante.

Desde el punto de vista estético, la tensión cultural inscrita en el nacimiento de estos discursos evidencia un arte asentado en numerosos préstamos culturales, tanto propios como ajenos, que lo caracterizan como un discurso "híbrido" (Carrasco, I. 1991). Muestra de aquello es la incorporación, en el mismo espacio textual, de diversos soportes, técnicas y diseños tanto de los cánones estéticos occidentales como de los cánones estéticos de la cultura mapuche ancestral; afirmando, por una parte, la continuidad del proceso de adopción cultural del arte tradicional platería, cerámica, textil, tallado- en la dimensión intercultural de la que forma parte, y, por otra, agregando una característica singular, respecto de la expresión tradicional, la que se encuentra en la posición de resistencia y de recuperación cultural que introduce como función de la obra de arte, debido a la permanente actualización que establece con el conflicto histórico y con el discurso hegemónico.(Cfr. García, M.2007, 2009, 2010).

Desde el punto de vista epistemológico, este arte enfatiza una función metacognitiva del discurso al inscribir un sujeto subordinado que habla y actúa desde la transculturación como un estado de permanente conflicto, lugar donde acontece el deseo de la recomposición cultural. Aquí el arte se transforma en un medio estratégico para reescribir y/o denunciar los acontecimientos y atropellos históricos, reflexionar sobre el proceso identitario y adoptar posiciones sobre la relación intercultural - lo anterior, particularmente inscrito en la poesía, la plástica y la música-; o para reflexionar sobre sus propios niveles de coherencia en los compromisos como pueblo -situación que inscribe estratégicamente en el "comics"-; y recuperar los elementos y categorías de la cultura tradicional ancestral -objetivo que atraviesa todas las expresiones artísticas-.

\footnotetext{
6 “...retradicionalización define básicamente el proceso por el cual se ponen en relación intertextual fragmentos provenientes de contextos anteriores con una finalidad explícita de producir un cambio. De esa manera la "retradicionalización" se plantea como distinta del proceso de "tradicionalización"; éste es constitutivo de todo fenómeno de transmisión cultural, mientras que la retradicionalización abarcaría instancias activas de diálogo con el pasado con fines políticos". (Fischman 2004. 140)
} 
En este complejo proceso socio-cultural, en la construcción de un sistema artístico autónomo con una identidad propia y diferenciada(García, M.2007), se hace presente un problema radical que afecta sus fundamentos ontológicos: distinguir "la singularidad"; situación de reconocimiento de la obra que en el presente sólo se plantea a nivel temático-cultural al atribuirse a la obra de arte lo "mapuche" porque en ella se hace evidente un "tema" asociado al ámbito de los elementos culturales o interculturales de la cultura mapuche junto a la explicitación de una identidad étnico-cultural declarada por el mismo artista; lugar de una enunciación ya conflictuada actualmente al surgir voces disidentes entre los artistas quiénes reivindican la calidad y el reconocimiento de la obra por la dimensión artística universal que alcanza ésta y no por una identidad étnico-cultural positivamente discriminada al amparo de los movimientos de derechos humanos, por ejemplo.

Más dificultoso se torna lo anterior si se considera el prolongado proceso de transculturación que ha afectado a los miembros de esta cultura, donde los parámetros de identificación antes enunciados, para el reconocimiento del "arte mapuche actual", resultan ser todavía más difusos para dar solidez a un sistema artístico en formación; al respecto si tomamos como referente sólo la variable de "manejo de la competencia cultural tradicional ancestral" que tienen los artistas mapuche-idioma, prácticas ceremoniales, reconocimiento del küpalme, entre otros-, ésta resulta ser bastante heterogénea, lo que incide fuertemente en varios sentidos en las mismas estrategias textuales.

En el marco de estas interrogantes establecer los fundamentos de un sistema artístico autónomo es todavía relativo, sobre todo porque se carece del conocimiento profundo sobre la actividad creativa del pueblo mapuche -donde se encuentran las producciones artísticas tradicionales y actuales-. En esta dirección, en este campo del conocimiento todavía son inciertas las respuestas a interrogantes fundamentales, como: ¿cuáles serían las bases estéticas de la cultura mapuche tradicional? -más allá de las generales que algunos estudios vinculan entre los pueblos indoamericanos-, y ¿cuáles de manera específica el artista recupera e inscribe en el nuevo espacio textual?, y ¿en qué grado éstas se encuentran presentes en una obra mediada tanto por los cánones occidentales como por los mapuche ancestrales?

Por otra parte, hipotéticamente, si podemos de alguna manera afirmar la existencia de bases o criterios estéticos en la cultura mapuche tradicional éstos pueden haber sido desplazados por diferentes motivos a un estadio secundario por los procesos históricos, como son los de orden hegemónicos que buscan socavar aquellos elementos y categorías - de diverso orden- de la cultura subordinada, lo cual nos pone frente a un escenario todavía más crítico, dado que si estas bases desaparecen estamos definitivamente frente a un cambio radical de un pueblo y su cultura. El sentido común nos señala que esta última sospecha no procede, dada la fuerte revitalización cultural, pero asumimos que nos encontramos a lo menos con desplazamientos y que estos son en variadas direcciones donde el más significativo es aquel que invisibiliza estas bases estéticas del reconocimiento del sujeto intracultural. A modo de reflexión, así como la cultura occidental alude normalmente a "lo bello" como categoría" estética articuladora de la sensibilidad heredada de la cultura greco-latina, donde "lo feo", "lo cómico", "lo trágico" o posteriormente "lo grotesco", "lo obseno", "lo fantástico", entre otras, miden el gusto; estamos claros de que los procesos de dominación también las han extendido como códigos a las culturas dominadas, donde pasan a ser inscritas en las prácticas artísticas suscitando un problema de hibridez cultural más complejo que aquel del valor de lo artístico en la obra.

En este marco de reflexiones, este trabajo inicial propone a discusión algunas bases estéticas de carácter provisorias de acuerdo a los principios de una "pragmática estética" (cfr. Romeu, V.

\footnotetext{
${ }^{7}$ El concepto de 'categoría' refiere a un elemento de clasificación, abstracto y codificado, que está sustentado en las cualidades de un objeto. Así una categoría (estética, artística, científica, técnica o filosófica) señalará y demarcará por convención una clase de objeto por sus cualidades, por sus características, substancias, esencias, atributos y propiedades. Es pertinente acotar, además, que cuando un concepto se nombra como categorial aspira a ser universal y deviene a-histórico; o dicho de un modo más simple: se cristaliza. (Cfr.García, Ana Claudia)
} 
2008), que Juan Acha (1999) siguiendo los argumentos de Bourdieu (2003) plantea de la siguiente manera:

"Las categorías o sentimientos estéticos son, pues, productos humanos o lo que es lo mismo, productos de la capacidad o facultad humana de sentir (la sensibilidad o el gusto). Pero al mismo tiempo son productos culturales en tanto las facultades humanas son potencialidades que se concretan en la realidad histórica y social...Lo artístico también es un producto cultural, pero derivado de lo estético en su calidad de concreción de la facultad denominada sensibilidad o gusto"(1999.34)

Ahora bien, si adoptamos como criterio general, según este autor, que las categorías estéticas no se transforman en el tiempo, sino que ellas adquieren distintas concreciones e intensidades en las obras de arte dependiendo de los períodos históricos(1999.39), señalo que los valores estéticos para la cultura mapuche se encuentran integrados a las múltiples dimensiones de la vida cotidiana y sacralizada de la cultura como un complejo tejido de significados y sentidos que organizan y dinamizan la cosmovisión mapuche tradicional, y que éstos trascienden las transformaciones históricas que ha debido soslayar la cultura, por lo cual se encuentran inscritos en las actuales producciones artísticas reforzando un arte con identidad propia y diferenciada.

Debido al carácter holístico o integral de la cultura mapuche ancestral no hablo de "categorías estéticas" como han sido propuestas en la lógica occidental, sino que propongo inicialmente hablar de "bases estéticas" para dar cuenta de ciertos "dispositivos nucleares" que organizan el quehacer creativo en la cultura y que sólo se vinculan y son explicables en el entramado de sentidos de la propia cosmovisión, por lo cual en la etapa que se encuentra esta investigación aquí se presentan con un carácter inicial y provisorio aplicado al mito y a su representación en diversos discursos artísticos, como la plástica y la poesía.

Propongo entonces hablar de: "Integridad", "fuerza", "simetría", "dualidad", como algunas de las bases estéticas que se explican al interior de aquello que se entiende como "el continuum cíclico" en la cultura occidental, pero que con más propiedad podría establecerse como "chiwvz" en la cultura mapuche, concepto que delimita la esfera epistemológica de la cultura y que integra dos dimensiones: "la conservación del equilibrio cíclico", y "la renovación cíclica" "El continuum cíclico" se explica aquí como la dinámica total de relaciones e interrelaciones que establecen los componentes del universo mapuche -"wallontu mapu"- al interior de una temporalidad en continuo movimiento que se dirige hacia el pasado y con ello afecta el sentido que tiene el presente y el futuro tanto en la vida cotidiana del hombre mapuche en el "nag mapu" como en la trascendencia de su linaje. Es al interior de esta dinámica que el hombre mapuche asume una vinculación ética con los antepasados y con las fuerzas o entidades que transitan desde y en las distintas dimensiones, reconociendo y legitimando este proceso en su "küpalme", potenciando la "fuerza" o "newen" en sí mismo y al interior del lof, con el fin de avanzar en el establecimiento de un espíritu sabio cumpliendo con las normas del "az mapu" y alcanzar con ello el retorno al "wenu mapu".

Este proceso para los hombres y para el mismo cosmos no está exento de vaivenes, por lo cual constantemente todo cambio descompensa alguna fuerza o algún componente, de donde los seres humanos y también aquellos que velan por él buscan compensar los estados de desequilibrio. Esto genera el sentido que tienen las ceremonias y las prácticas socioculturales en general: el respeto al "az mapu"-.

De esta interacción dinámica del cosmos y de la persistente búsqueda de un estado de equilibrio de éste por parte del ser humano y de las fuerzas nouménicas, surgen las vías explicativas de la realidad y la ejecución de las prácticas socioculturales como su extensión comprehensiva, al interior de las cuales se originan los temas y/o tópicos de los discursos tradicionales y actuales dando cuenta del fortalecimiento de la norma o de su trasgresión y de su posterior rearticulación.

a) La Conservación del equilibrio cíclico: con esta dimensión refiero a la dinámica que concurre como instancia de comunicación y de intercambio en la relaciones interpersonales y comunitarias y que se puede plasmar en nociones tales como "trafkin"; dinámica que se 
actualiza ya sea en la actividad ceremonial de orden ritual dando origen a la negociación y compromiso con el o los ser(es) superior(es) y mediante el ofrecimiento e intercambio de los "newen" -fuerzas-, o en la vida cotidiana en las relaciones interpersonales o comunitarias a partir del intercambio o "negociación" como concepto de "no deuda". Este proceso es habitualmente simbolizado a través del mito de "txen -txen y kai kai vilu" donde se muestra el proceso de enfrentamiento de fuerzas, descompensación y recuperación del equilibrio cósmico, personal o colectivo; o en aquellos relatos donde se dinamiza el conflicto de las energías positivas y negativas y que repercute en evidenciar el origen y proceso de la enfermedad y el reestablecimiento de la fuerza vital.

b ) La renovación cíclica: Cuya dinámica se comprende en torno al concepto de tiempo y trascendencia a partir de la noción de "kuifi", en tanto todo sentido del "ser" se establece como un viaje permanente -por las dimensiones tangibles y nouménicas- de encuentros y reencuentros vinculados por el "Küpalme" -linaje-, compromisos, o por el "tugün" -territorio con un mismo ser tutelar-. Básicamente este proceso se encuentra en la "rememoración" o regreso al tiempo anterior para explicar y evaluar las condiciones del presente y del futuro, y se concreta en el arte en la activación de los lenguajes de los "pewma"-sueños- y de los "perrimontun"-visión- como avisos y guías desde la dimensión nouménica, además se simbolizan a través de la iconografía o a través de los tropos en la poesía, por ejemplo. Aquí también se hace necesario entender que se establecen los hitos referenciales a partir de ubicar ciertos acontecimientos relevantes para la cultura, y con ello marcar el inicio de otro comienzo: wetripantu, nguillatun, el nacimiento o muerte de una machi, un conflicto histórico, entre otros. De esta relación entre lo epistemológico y lo ético surgen las bases estéticas, expresadas por el momento como "Integridad", "fuerza", "simetría", "dualidad".

Mientras la "integridad", refiere a la representación completa del todo o de una parte de él de manera unitaria cada vez, a través de procedimientos retóricos como la sinécdoque, la metáfora o el símbolo, entre otros; esta base estética alude a una mirada siempre relacional de la realidad y de la interacción al interior de ella, ya sea evidentemente explícita o de manera asociativa respecto de un contexto -común- que completa los significados -introducidos en la obra-.

La "fuerza", refiere a la capacidad del ser de hacerse visible mediante la territorialización de hecho o simbólica que provoca su energía, y se activa en su sucesivo autodescubrimiento respecto de su linaje y territorio, su afirmación en el rol comunitario, y es colaborativa entre varias entidades, lo que permite además la diferenciación y afirma la singularidad individual y/o colectiva.

La "simetría", refiere a la representación del orden de los elementos, seres o entidades en una coordenada espacio-temporal doble -simultáneamente imbricada con lo nouménicodesde el estado de equilibrio/poder como atributo ideal del ser o del ente.

La "dualidad", es el establecimiento de la co-presencia como elemento de interacción, en un estado dinámico del cosmos y que busca alcanzar el equilibrio mediante la conjunción de sus energías, y se actualiza en lo dialógico en el ámbito comunicativo, en el cohabitar junto a otros -tanto en el plano visible como en el no visible, y entre éstos-, en lo masculino-femenino, positivo-negativo, en el intercambio de energías, en la composición de las energías en un mismo ser o entidad, entre otros.

Seguramente son muchos otros los referentes estéticos de esta cultura, sin embargo para efectos del presente trabajo mi interés es mostrar cómo estas bases estéticas se encuentran integradas al arte mapuche actual a través de la representación que realizan los artistas sobre el mito tradicional, particularmente del mito de Txen Txen y Kai Kai.

El mito como parte del proceso de retradicionalización del arte mapuche actual, es un dispositivo epistémico a través del cual transitan múltiples significados y sentidos; es, por lo tanto, un lenguaje complejo que puede describirse como una matriz simbólica en la cual se desplazan y actualizan elementos y categorías fundamentales del saber cultural tradicional y ancestral. 
Si el proceso de retradicionalización "supone una instancia de búsqueda en el pasado, una indagación en fuentes a las que se considera "poseedoras de un saber anterior" para, a partir de ahí, reconstruir una tradición." (Fischman, F.2004.138-139), el mito para el arte mapuche actual se convierte en uno de los medios más poderosos para recuperar no sólo significados sino aquellas bases estéticas que le son propias y posicionarlas gradualmente en los actuales proyectos artísticos, a partir de una estrategia que desde mediados de esta década busca desplazar de la obra los elementos, soportes y técnicas de la producción artística y del saber occidental, para convertirlos en significantes vacíos y en su lugar enfatizar y territorializar los propios.

Teniendo presente, entonces, que nos encontramos con una vinculación indisoluble entre estos dos ámbitos, el arte mapuche actual refiere una amplia panorámica sobre la relación que se da entre el ser humano y los seres antropomorfos; sin embargo, exceptuando el textil y la platería moderna donde tradicionalmente se expresan símbolos del "mito cosmogónico", en veinte años de expresiones artísticas sólo surgen algunas referencias esporádicas a este relato, particularmente a través de la poesía de Elicura Chihuailaf y del audiovisual de Sofía Painequeo "De porqué nos llamamos mapuches".

En este volver hacia el tiempo anterior otros mitos se constituyen en el centro de referencia, principlamente el mito de "Txen Txen y Kai Kai", un relato que se encuentra habitualmente asociado al mito del diluvio cuando Kai Kai, serpiente del agua, busca la extinción del pueblo mapuche debido a las desobediencias de éste a las normas ancestrales, mientras Txen Txen busca salvarlos subiendo los cerros hasta refugiar en ellos a un hombre y a una mujer vieja y a un hombre y una mujer joven, la familia divina, quienes dan origen a la segunda generación del pueblo mapuche.

En este relato, señala el wülzugufe ${ }^{8}$ Juan Ñanculef "Se plantea... la lucha de dos energías en oposición necesaria, energías que están representadas por dos serpientes que luchan por muchos ciclos en oposición permanente, pero no para destruirse una de la otra, sino para lograr un equilibrio necesario, permanente y cíclico. La energía que representa la serpiente TxegTxeg, es ni mas ni menos que la tierra, y la energía que representa la serpiente Kay-Kay es la del Agua" (2009.6)

Una de las primeras representaciones artísticas que hace referencia a este relato se encuentra en la pintura, particularmente en el arte muralista, tal como lo podemos apreciar en el mural realizado el año 1997, por los artistas plásticos Doris Huenchullán y Eduardo Rapimán, en la ciudad de Temuco (Chile).

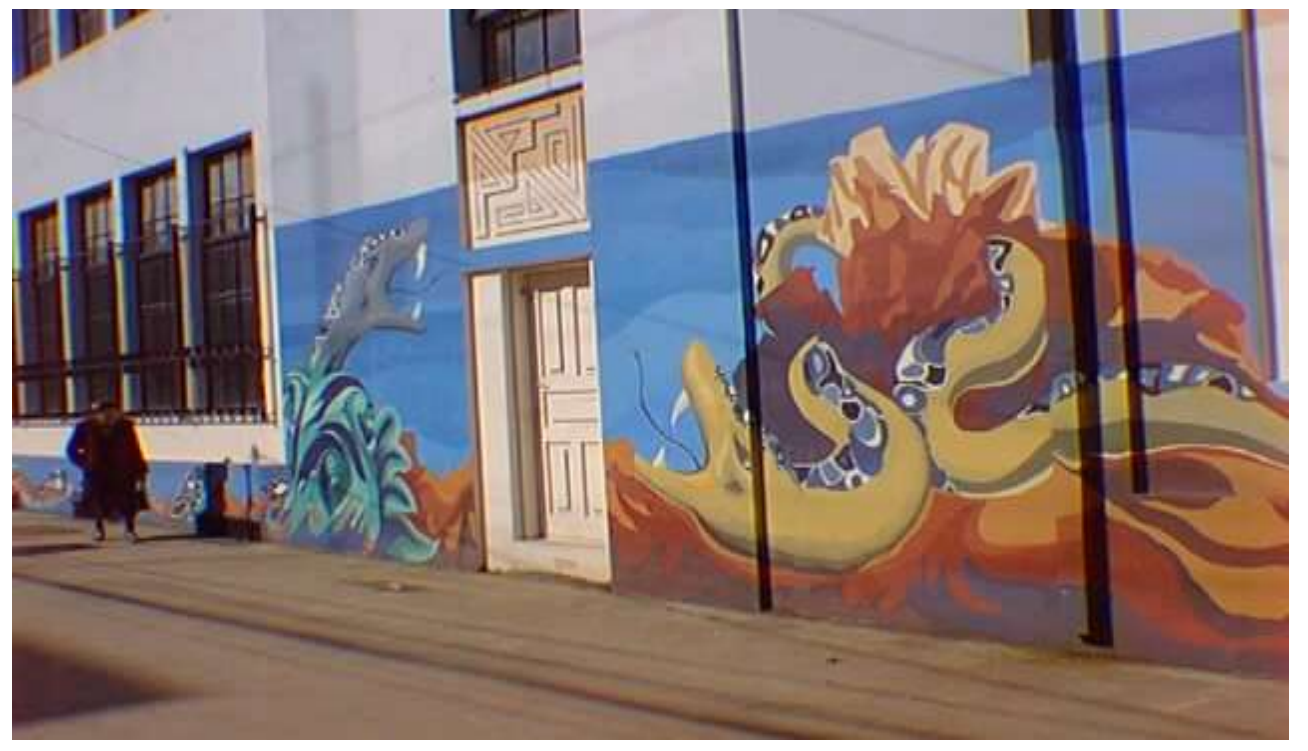

\footnotetext{
${ }^{8}$ Dador de conocimiento.
} 
Como se hace visible en esta propuesta, los criterios estéticos aquí se encuentran dinamizados como parte de la lectura intracultural, así la "integridad" surge en la convergencia que tiene todo elemento formal y semántico desde y hacia una idea totalizante sobre lo que es lucha telúrica de estas dos fuerzas, sustentada en una estrategia visual que dispone ambas figuras moviéndose hacia el enfrentamiento. Por otro lado, el logro del componente "fuerza" se hace visible por la territorialización omnipotente y homotópica de cada una de las culebras como "dueña" o "ñgen" de sus respectivos territorios, el cual es sugerido a partir de la disposición de los colores; un topos que domina con su energía al ser parte de él y que se muestra en la intensidad de su gesto de defensa y dominio. La "dualidad" les es inherente en la medida que ellas representan la dinámica permanente de fuerzas en pugna -tierra y agua- que en los distintos ciclos cosmogónicos se equilibran/desiquilibran, una dualidad siempre en movimiento en cuya representación no están significados los seres humanos porque aquí lo relevante es el conflicto, la desestabilización, una instancia de complejo simbolismo que señalo más adelante. Por último la "simetría" pone al amparo un cierto orden que se juega, por una parte, por dos puntos exactos de desplazamiento hasta un punto medio de convergencia, y, por otra, es la reunión entre la estaticidad/movimiento, explicadas como la captura del movimiento mostrado en la posición de ataque/defensa sin llegar a tocarse nunca y la dinámica de dos figuras que surgen desde los márgenes de la pared, sobre las cuales fueron pintadas, para ir a la confrontación en ese mirarse y comunicarse sin ceder territorio.

Otra obra mural con este motivo es la del artista plástico, músico y escultor Christian Collipal, quién realiza esta producción el año 2004 para una institución pública en la ciudad de Temuco (Chile).

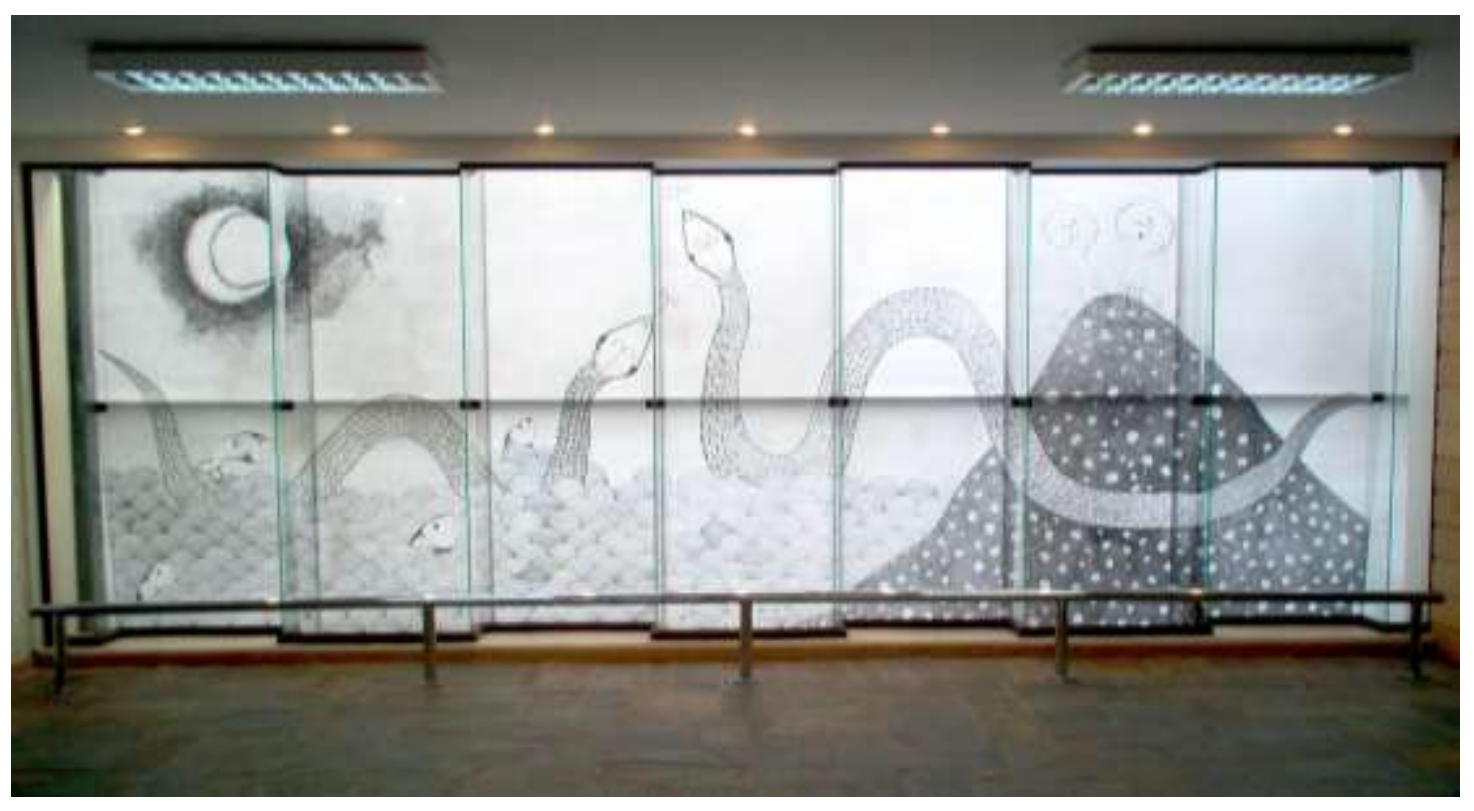

A diferencia de la anterior representación, esta obra incluye la dimensión humana graficada en las dos figuras que se encuentran sobre el cerro, a partir de una narrativa del conflicto telúrico que se inscribe justo en el momento posterior al ataque de Kai Kai y a la lucha frontal entre ambas serpientes; aquí es Txen Txen quién avanza sobre las aguas haciendo retroceder a Kai Kai con el fin de resguardar la vida humana. Es el relato mítico en su "integridad", que narra la subida de los cerros y el retroceso de las aguas, espacio en el cual se debaten elementos significativos -los "peces", como vida/alimento que asoman desde la profundidad, y las "piedras", como fuerza/poder que sostienen a los cerros y a los hombres, mientras todo lo anterior se muestra en el descentramiento cósmico evidenciado en la fusión de una luna/sol oscurecida y el horror en los rostros de los hombres al contemplar el caos. 
Por otra parte, la "simetría" se representa a partir de una doble condición: la estrategia del peso visual y el estadio compensatorio de las fuerzas en pugna, ambas imbricadas para soportarse en el balance de la lucha por el dominio.

¿Qué hay además en la lectura de estas obras visuales? Más allá de los señalado para este relato, y en la orientación de que toda lectura del mito en un articulador semántico fundamental para la cultura, las figuras de Txen Txen y Kai Kai conllevan un sentido profundo en su representación que las relaciona con las características que tienen muchas figuras totémicas de los diversos territorios mapuche: el cómo dinamizan el mundo a partir de la concepción dual de las fuerzas y cómo actualizan los ciclos humanos y divinos a través de los acontecimientos en que se inscriben cada vez.

Desde el punto de vista totémico, como hemos aludido antes, cada territorio mapuche se entiende descendiente de un linaje protegido por el espíritu del ancestro que da origen al lof, además de un espíritu protector con quién formó alianza; esta última figura normalmente es dual, como lo vemos representado por la artista plástica Lorena Lemunguier.

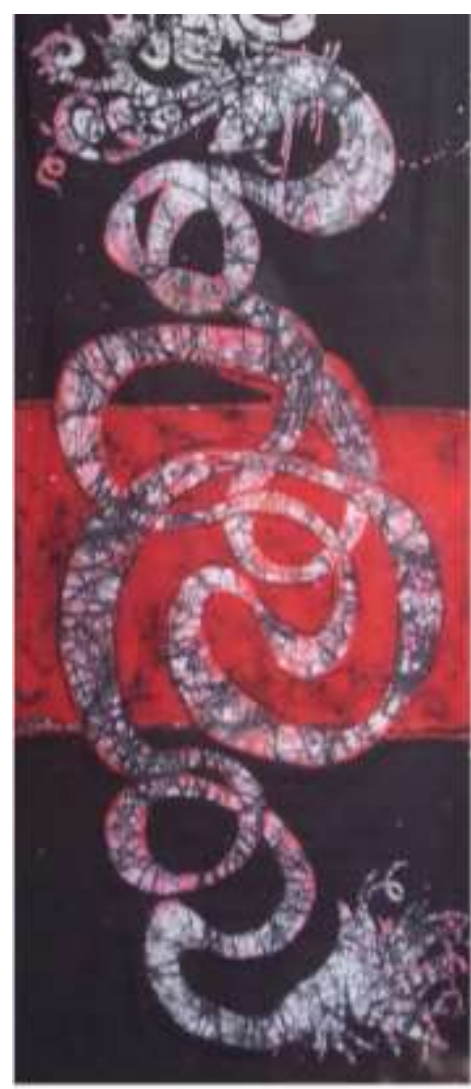

Texto 1

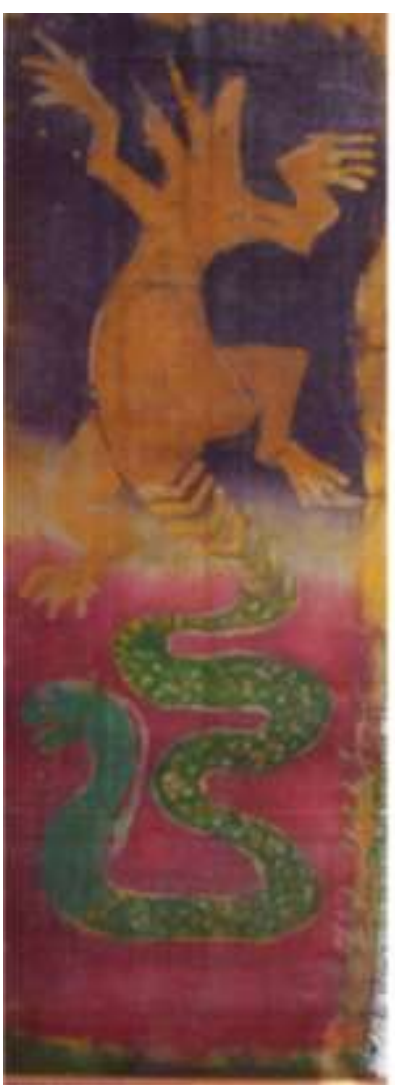

Texto 2

Realizadas en tela con la técnica "batik", para Lemunguier, de forma similar a Txen Txen y Kai Kai -texto 1- se encuentra "Ngürufilv" o zorroculebra -texto 2-, un relato que corresponde a los denominados "mitos de transformación", si aplicamos la propuesta de Carrasco.

Ya sea para el primero como para el segundo, en ambos casos lo expresado corresponde a una concepción dual de los seres antropomórficos y en cuya representación se encuentra cifrada metonímicamente la oposición de "fuerzas" que sostienen el equilibrio del universo, ya no entendidas como dos individualidades que mantienen una lucha frontal sino como una sola "fuerza" que se metamorfosea en dos entidades que tienden a mirar en sentido contrario y por lo tanto a dirigir sus energías hacia los polos inversos; así también se inscribe la oposición entre estaticidad y movimiento, debido a la posibilidad de actualizar el dominio de una sobre la otra 
lo que provocaría el desbalance cíclico. Sin embargo, en la concepción de Lemunguier, estas figuras más bien adquieren una cierta sintonía y sincronía entre sí y con su contexto, lo que se traduce en los desplazamientos ondulantes y replegados -no tensionados-que mantienen y que generan una disposición a la interacción y por lo tanto al equilibrio, proponiendo un énfasis en la "simetría" como el estadio ideal de las fuerzas.

Las lecturas anteriormente realizadas sobre el mito de Txen Txen y Kai Kai evidencian la dinámica de las grandes trasgresiones y también de las grandes alianzas al interior de la nag mapu, y éstas refieren a los modos de establecer los períodos -de tiempo- al interior del círculo permanente de la vida en el mundo mapuche, dando lugar a aquello que se reconoce como "ciclos".

Desde la lectura de trasgresiones, debido a las permanentes irrupciones e imposiciones de orden hegemónicas con que se instala occidente en el territorio mapuche inscribiendo ideologías y por lo tanto conductas opuestas a la norma ética ancestral, los actuales artistas mapuches adoptan como referente la lucha de Txen Txen y Kai Kai para desplegar un significado que va más allá del mito del diluvio, representando a través de ellas "las etapas del conflicto mapuche". Establecidas cuatro etapas hasta el presente, la primera se vincula efectivamente a la lectura del "mito del diluvio" mientras las restantes son desplazadas entre aquella y el presente histórico; así, el segundo enfrentamiento conlleva la lectura ética que tiene lugar con la intervención de occidente en el mundo mapuche ancestral a partir de la llegada de los españoles; mientras la tercera, refiere al período de la formación del estado nación chileno ante el cual este pueblo se alza en guerra; y por último, la cuarta, alude a la actual situación histórica que vive la cultura mapuche, donde muchos de sus miembros han abandonado sus tierras para emigrar a la ciudad, perdido el idioma y las prácticas ancestrales aceptando las normas de la sociedad chilenaoccidental y actualmente también de la sociedad globalizada. Este desarraigo obligado por los procesos hegemónicos, se ve como motivo de enfermedades y también de descentramiento del "ser" -espíritu, alma, cuerpo- y como pérdida de fuerzas del mismo "wallontu mapu" -la tierra mapuche-, proceso que explica la lucha que se tiene actualmente por recuperar lo propio, entre éstos la recuperación del territorio.

La pintura en cuero del artista plástico Juan Silva Painequeo ha sido tal vez la más explícita con este tema, desarrollando con detalle el proceso de los ciclos de la historia mapuche, pero desde una lectura que instala su obra en la articulación de la "fuerza" originaria o trascendente de este pueblo, haciendo ver que este conflicto es primero y ante todo una lucha a nivel de "fuerzas" o "newenes". Desde este punto de vista, el conflicto de Txen Txen y Kai Kai representa no sólo el acontecimiento histórico, sino que mantiene un trasfondo de carácter espiritual donde lo que se debate es el cuidado -o no- de una forma de conocimiento o "kimun"; resguardo del conocimiento ancestral que es acceso a una forma de "poder" y que históricamente su dominio ha permitido formar las alianzas necesarias para la protección del mismo pueblo mapuche, o la potenciación y expansión de su fuerza al interior de su territorio. 


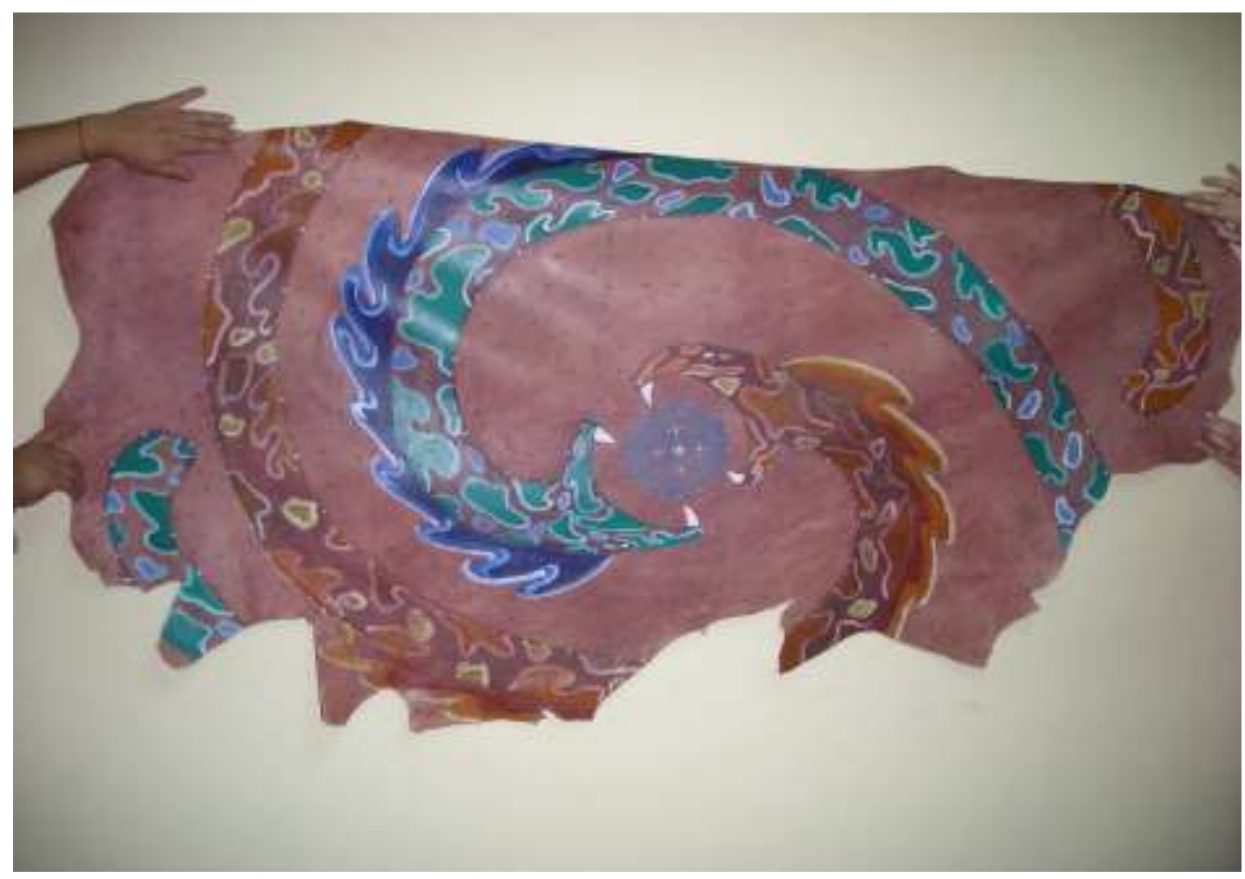

Tal como lo vemos en esta obra en particular, Juan Silva Painequeo se juega por una representación que privilegia el sentido de la trascendencia de Txen Txen y Kai Kai en razón de su dominio sobre las fuerzas telúricas y cósmicas, y esto se fija en la obra a partir de la proposición de "simetría" que realiza como el estado justo de equilibrio/poder en tanto atributo ideal del ser, una relación entre Txen Txen y Kai Kai que se mantiene precaria y equitativamente al sostener en su dinámica cíclica el elemento cósmico. Un movimiento siempre en sentido contrario al reloj y en orientación al sentido de rotación de la tierra, el que además se homologa como la vuelta al tiempo anterior desde el punto de vista mítico-simbólico. Constituyéndose este mito en un relato fundamental para la recuperación y la transmisión del saber cultural tradicional del pueblo mapuche, éste no sólo es actualizado por los textos visuales como hemos visto anteriormente, sino además por otras formas expresivas como el teatro, el audiovisual y también por el discurso poético, donde surge la figura de Txen Txen y Kai Kai vinculada al tránsito de un ciclo a otro, invocación a un tiempo de estabilización del pueblo mapuche, aspiración a la libertad y a la autonomía:

"iLevante Wiyiche! Dice ahora Ponciano Rumian y esgrime/ con su banjo esta mañana.../ ¡Levante Wiyiche! -me dice-/ y toca y canta.// ¡Levante! responde Arturo Camiao/ y cruza por estas pampas dejando huellas en el pasto/ en la escarcha de los hualves...//...En este solsticio de invierno/ marchamos a los montes que se abrazan llorando.../En la gran roca...nuestra memoria./el taita Wenteyao vigila el mar/ xren xren...xren xren... / se escucha en nuestros corazones." (César Millahueique. Oratorio al señor de Pucatrihue)

La poesía de César Millahueique, al ubicarse en el actual momento histórico del pueblo mapuche abre el espacio textual a la reflexión sobre los procesos de transculturación y a la pérdida de la memoria cultural, lugar donde de manera transversal a otras poéticas inscribe su poesía en el ámbito de los temas de la resistencia cultural, de la reafirmación de la identidad étnico-cultural, y de la revisión histórica. En este sentido, "Oratorio al Señor de Pucatrihue" (2004) o "taita Wenteyao", representa un mundo circular primigenio a partir de la oración del rito de nguillatun -ceremonia sagrada de agradecimiento y protección de Küme Fütra Pvllv-, en el cual a través del "pewma" (o sueño) el emisor textual evalúa los procesos de dominación cultural, el despojo de las tierras y busca la protección de los espíritus tutelares, entre los cuales invoca este relato mítico. 
Así, homologando el sonido del "kultrung" (o tambor ceremonial) a través de la disposición fónica y rítmica de los versos: "xren xren ...xren xren.../se escucha en nuestro corazones", Millahueique alude al llamado que los Wilyiches hacen a la orilla del mar al Taita Wenteyao en la ceremonia del nguillatun, donde mar y tierra son una y la misma "fuerza".

De manera similar al sentido que adquiere este relato en Millahueique, la poetiza Maribel Mora Curriao en su poema "Malos sueños" abre el espacio textual para referir el presente histórico de la cultura a partir de la experiencia personal. Con una estrategia textual que divide el poema en dos partes: una en prosa donde ubica los antecedentes familiares y otra en verso donde proyecta el futuro, el hablante en esta segunda parte advierte sobre sí signos asociados a la pobreza -"como quilas florecidas"- y a la muerte -"graznidos nocturnos"- como condición sine qua non por el desplazamiento territorial al que se ve obligada esta familia. Proyectado este acontecimiento de manera similar y circular a través del linaje, visualiza a Treng Treng y Kai Kai como la única esperanza para alterar lo ya fijado por el acontecimiento histórico: el inicio de un nuevo ciclo.

\begin{abstract}
"I...
Como quilas florecidas/ o graznidos nocturnos/ pasan los sueños/ que formaron mi costado/amargos vaticinios/ en la memoria de la noche.// Triste fue el sueño de mi abuelo,/ soledad de nieve/ en las quebradas y en los huesos./ Triste el sueño de mi madre,/ oscura torcaza aleteando/ contra el viento.// Pero más triste aún/ el sueño de mis hijos,/ de los hijos de mis hijos/ en territorio de nadie.// Mañana poblarán la tierra/ las grandes sierpes de antaño:/ Treng Treng, Kai Kai/ y rugirá el cielo/ sobre nuestras cabezas.// Y luego/ habrá brotes de cerezo,/ entonces luna nueva,/ nuevos sueños habrá,/ mariposas en el horizonte.// Por ahora nada somos,/ ni siquiera paja/ en el ojo de Dios/ que nos olvida..." ("Malos sueños" 2007.332-3)
\end{abstract}

\title{
Lecturas de la recuperación cultural: las resignificaciones estéticas
}

El proceso artístico que lleva a cabo el pueblo mapuche en la actualidad es un proceso complejo que responde a situaciones históricas avasalladoras, desde las cuales resurge con "fuerza" territorializando espacios diversos a través de prácticas culturales innovadoras como son las prácticas artísticas, y según vemos en este trabajo manteniendo muchos componentes culturales tradicionales ancestrales como son los componentes estéticos. Una aseveración como ésta abre un espacio de discusión amplio donde se debe complementar y completar la propuesta realizada aquí, debido a que ella se ubica en los márgenes iniciales de un largo camino que hay que recorrer, no sólo porque debe ser estudiada a la luz de las expresiones tradicionales para ser coherentes con el origen de estos criterios estéticos, sino porque además debe encontrar su formulación en el idioma de origen donde las nociones sean explicadas desde la intraculturalidad, para en una segunda instancia ser traducidas al castellano.

Consciente de que este inicio tiene estas falencias y otras que sin duda surgirán en el tiempo, cabe asumir que es necesario y un desafío abrir esta puerta. Por una parte porque el sistema artístico en progresión adquiere visibilidad, solidez y legitimidad al diferenciarse de otros sistemas artísticos, y porque constituye un logro significativo para el arte latinoamericano. Desde este punto de vista, señalaré lo que ya expuse el año 2007: entender qué es la dimensión estética de la obra es distinguir los criterios de lo creativo en la dinámica cultural, donde se conjuga lo religioso con lo pragmático, lo expresivo con lo funcional -lo cual también ayuda a explicar la orientación práctica de ésta, de los proyectos artísticos y del sistema en progresión a la autonomía y su relación con la resistencia y reivindicación cultural-. (cfr.García, M. 2007. 2009) 


\section{Bibliografía.}

ACHA. Juan. (1999). Los conceptos esenciales de las artes plásticas. Ediciones Coyoacán. México. Pp.186.

BOURDIEU. Pierre. (2003). Creencia artística y bienes simbólicos. Elementos para una sociología de la cultura. Aurelia Rivera Grupo Editorial. Argentina.

CARRASCO, Iván. (1991). "Textos chilenos de doble registro". Revista Chilena de Literatura №37. Universidad de Chile. Santiago. Chile.

CARRASCO, Hugo. (1990). "La lógica del mito mapuche". Estudios Filológicos. №25. Facultad de Filososfía y Humanidades. Universidad Austral de Chile. Valdivia. Chile.

MORA CURRIAO, Maribel. (2007) La memoria iluminada: poesía mapuche contemporánea. Jaime Huenún Editor, CEDMA Ediciones. Málaga. España.

CHIHUAILAF, Elicura. (1999). Recado confidencial a los chilenos. LOM Ediciones. Santiago. Chile.

FISCHMAN, Fernando. (2004). "Procesos sociales y manifestaciones expresivas. Una aproximación a partir de los estudios folklóricos" Revista Potlatch. Cuaderno de Antropología y semiótica. Año $1 \mathrm{~N}^{\circ} 1$. UN editora. Buenos Aires. Argentina.

GARCÍA, Ana Claudia. (2004). "Puntuaciones sobre arte, tecnología y nuevas categorías estéticas" http://www.liminar.com.ar/jornadas04/ponencias/garcia.pdf.

GARCÍA, Mabel. (2007). "Estrategias del discurso artístico mapuche como proyecto de autonomía estético-cultural" en Memorias 50 años Flacso. Quito. Ecuador.

GARCÍA, Mabel. (2009). "Comunicación intercultural y arte mapuche actual” Revista Alpha. Universidad de Los Lagos. Osorno. Chile.

ÑANCULEF HUAIQUINAO, Juan. (2009). Conformación y estructura del territorio mapuche. Texto digital difundido por el autor a través de email

MILLAHUEIQUE, César.(2004). Oratorio al señor de Pucatrihue. La estocada sorpresiva. Santiago. Chile

QUILAQUEO, Daniel et al. (2005). Educación, currículo e interculturalidad. Elementos sobre formación de profesores en contexto mapuche. Facultad de Educación. Universidad Católica de Temuco. Temuco. Chile.

ROMEU, Vivian. (2008). "De la "estética trascendental" a la pragmática estética; esbozo de una teoría comunicativa del arte. Revista Observaciones Filosóficas. N ${ }^{\circ}$. Universidad Complutense de Madrid. España.

http://www.observacionesfilosoficas.net/delaestetica.html

TOLEDO LlanCAQUEO, Víctor. (2006). Pueblo mapuche. Derechos colectivos y territorio. Desafíos para la sustentabilidad económica. LOM Ediciones. Santiago. Chile. 